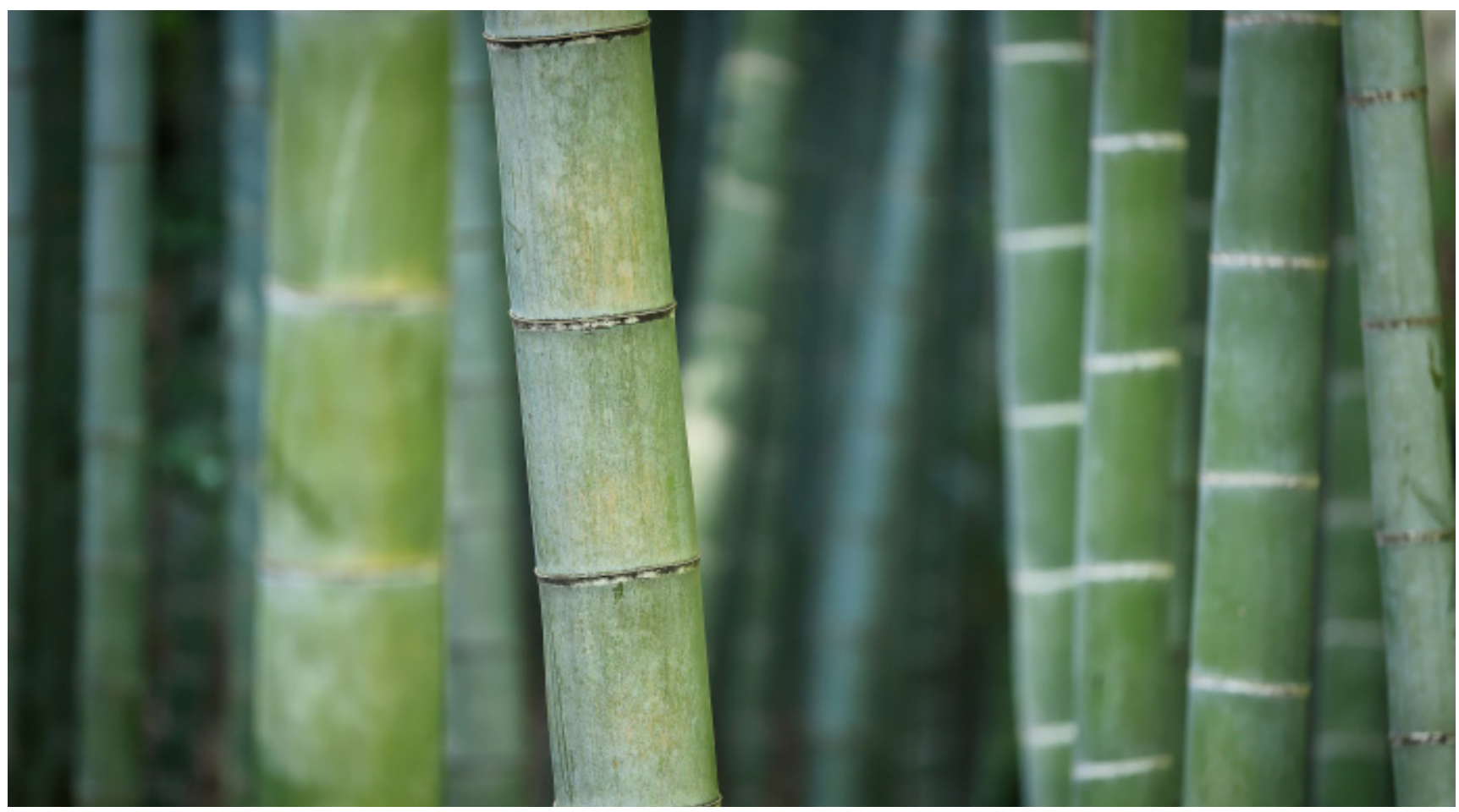

\title{
Análisis del comportamiento del guadua "BAMBU" sometido a postensado
}

\section{Behavior Analysis of Guadua "BAMBU" Subjected to Post-tensioned}

María Fernanda Granados-Páez

Ingeniera Civil, mariafernandagpgrp@ufps.edu.co,

https://orcid.org/0000-0002-6943-5219, Universidad Francisco de Paula Santander, Cúcuta, Colombia

Roberto Bohórquez-Rojas

Ingeniero Civil, robertobohorquez@ufps.edu.co,

https://orcid.org/0000-0003-0019-1057, Universidad Francisco de Paula Santander, Cúcuta, Colombia

Ender José Barrientos-Monsalve

Arquitecto, enderjosebamo@ufps.edu.co,

https://orcid.org/0000-0001-6673-0223, Universidad Francisco de Paula Santander. Cúcuta, Colombia

*Autor de correspondencia: mariafernandagpgrp@ufps.edu.co 


\begin{abstract}
Recibido: Febrero 17 de 2019
Aceptado: Mayo 25 de 2019

Cómo citar: M.F. Granados-Páez, R. Bohórquez-Rojas y E.J. Barrientos-Monsalve, "Análisis del comportamiento del guadua "BAMBU", Sostenibilidad, Tecnología y Humanismo, vol. 10, no. 2, 49-55, 2019.
\end{abstract}

Resúmen

El siguiente trabajo pretende dar a conocer el comportamiento de las propiedades de bambú sometido a postensado, a través de una investigación científica que tiene como objetivo general Aplicar ensayos de laboratorio al bambú o guadua angustifolia kunth sometida a postensado a distintas cargas axiales a lo largo de su luz. La investigación tiene un enfoque cualitativo; de la misma manera, es de carácter documental ya que se puede realizar a nivel exploratorio, descriptivo $o$ explicativo estudiando variables independientes a partir de datos secundarios, con análisis de estos datos secundarios se puede elaborar importantes conclusiones relacionadas con el comportamiento o estado de las variables; para el análisis de los resultados se utilizaran instrumentos como fichas, computadores, y sus unidades de almacenaje, cuadros de registros. El mismo está estructurado de la siguiente manera en un primer bloque se presenta el planteamiento del problema, los objetivos del estudio y la justificación. En un segundo bloque todo lo referente a la metodología, se desarrolla el tipo y diseño de la investigación, las técnicas de recolección y procesamiento de la información. Finalmente se presentan las conclusiones.

Palabras clave: Angustifolia, Bambu, construcción, guadua, postensado.

\section{Abstract}

The following work intends to present the behavior of the properties of bamboo submitted to posttensioning, through a scientific research that has as general objective To apply laboratory tests to bamboo or guadua angustifolia kunth submitted to post-tensioning to different axial loads along its span. The research has a qualitative approach; in the same way, it is of documentary character since it can be made at exploratory, descriptive or explanatory level studying independent variables from secondary data, with analysis of these secondary data important conclusions related to the behavior or state of the variables can be elaborated; for the analysis of the results instruments like cards, computers, and their storage units, tables of registries will be used. It is structured in the following way in a first block presents the approach to the problem, the objectives of the study and the justification. In a second block, everything related to the methodology, the type and design of the research, the techniques for collecting and processing the information are developed. Finally, the conclusions are presented.

Keywords: Angustifolia, Bambu, construction, guadua, post-tensioned. 


\section{Introducción}

Desde tiempos inmemorables el hombre del trópico ha hecho provecho de los múltiples recursos naturales que el planeta nos regala, especialmente de las diferentes especies locales de bambú, como materia prima para la construcción de sus casas sus casas, sus balsas, sus puentes, sus armas, herramientas y su comida. Siendo también una de las plantas típicas del hábitat tropical litoral en África oriental, que conforma la cuna del hombre, se puede asumir que el bambú forma parte del primer kit de herramientas de la humanidad [1].

Por consiguiente entre de las más de 1400 especies reconocidas de bambúes, son plantas que habitan las zonas tropicales, aunque pueden crecer bien en climas templados, viven silvestres desde el nivel del mar hasta las altas montañas nevadas. La mayoría de los bambúes prefieren habitar en lugares húmedos y conservados, donde la presencia de neblinas es frecuente, o bien en las orillas de arroyos y ríos, también existen especies que viven en lugares secos la mayor parte del año y que solo obtienen el agua en los pocos días de lluvia de determinada región. En los países de Asia los bambúes son plantas bien conocidas, pues se han sido utilizadas por el hombre desde tiempos remotos. Hay bambúes silvestres en África y en casi todos los países de América con excepción de Canadá [2]. Por otra parte, los bambúes pueden crecer en extensas poblaciones donde son los únicos vegetales viviendo o bien como individuos solitarios formando parte del bosque o la selva. Las islas del caribe tienen bambús silvestres y también los hay en el este de los Estados Unidos. Australia cuenta con tres especies nativas y las islas del pacífico también tienen bambúes. Cabe agregar que el bambú es un recurso natural que ha sido aprovechado intensamente por el hombre durante milenios en las regiones más populosas de la Tierra. La planta es común a todas las Regiones tropicales; en algunos continentes se extiende al norte y al sur de los trópicos de Cáncer y Capricornio [3].

Es importante resaltar que el bambú se adaptó a las condiciones ecológicas diferentes del desde las culturas más antiguas en el oriente, con mayor importancia en los países de China, India y Japón. Esta gramínea ha servido para una gran diversidad de artesanías, e incluso sirve a manera de enchapes para pisos, como papel, como alimento, etc. En el continente americano ha sido utilizado desde el periodo precolombino aprovechado de un modo empírico por las grandes cualidades que posee este material, su bajo peso y costo, su rápido crecimiento y excelentes cualidades a tracción, flexión y compresión [4]. Este material está revolucionando cada día más por su rentabilidad ya que su crecimiento es muy rápido y se da en cualquier zona tropical y no es necesario un cuidado tan arduo como otro tipo de material y esto lo hace un material innovador e ideal para la industria.

También en Colombia, la producción de materiales para la construcción es muy importante, ya que cubren las necesidades no solo de la región de Caldas y las fincas y poblaciones del valle del Cauca, sino también de la ciudad de Bogotá y otros centros urbanos [5]. En la ciudad de Manizales destacan muchos buenos ejemplos de arquitectura vernácula fabricada con guadua. Debido a la problemática enfrentada es hacer de este materia un material esencial para la construcción de todo tipo ya sea desde casas que ya actualmente se fabrican hasta puentes y edificios que nos permita tener un sistema auto sostenible amigable con el medio ambiente para ello queremos en este proyecto determinar la resistencia al pos tensado de la guadua o bambú y determinar si es un material ideal para una magnitud de construcción superior a la de una vivienda. El bambú es conocido como la planta de mil 


\section{2}

usos, esta maravillosa y majestuosa planta es La construcción en los últimos años ha versátil, flexible y de gran dureza, apta para tenido un desarrollo muy alto en cuanto la ser utilizada en diversos campos tales como tecnología y diversificación de la misma; construcción, jardinería, pesca, artesanía, tacando el problema que se posee a la hora muebles, utensilios, así como el ser fuente de diversificación, el cual es no contar con comestible y otros usos inimaginables [6].

las herramientas para realizar un diseño con el fin de construir en el material deseado [9].

Por tanto el propósito principal fue aplicar Este trabajo aportará a la ingeniería civil y sus ensayos de laboratorio al bambú o guadua angustifolia kunth sometida a postensado a distintas cargas axiales a lo largo de su luz, para analizar el comportamiento del bambú a la aplicación de distintas cargas según su estado de madurez. Antes y después del postensado y registrar los resultados que se obtuvieron antes y después del postensado del bambú para una mejor utilización y determinar si este material puede ser utilizado para la elaboración de puentes de uso vehicular.

En este trabajo se realizará un estudio preliminar de la resistencia al pos tensado del bambú o guadua angustifolia para determinar por medio de una serie de ensayos a comprensión y/o tensión, utilizando probetas de guadua de diferentes dimensiones la aplicación de cargas que permitan llegar a unos resultados específicamente relacionados con el contenido de humedad, la densidad, el módulo de elasticidad, y resistencia a la compresión paralela y perpendicular a la fibra, resistencia al corte paralelo a la fibra, Y no se permitirá la construcción de puentes a la tensión y a la flexión, para analizar si o estructuras diferentes de las edificaciones este material se comporta idealmente para de uso de comercio, industria o educación darle un uso más importantes en la industria [11]. Por otro lado tendría un gran aporte a la de la construcción, considerado por [7], para sociedad ya que ayudaría a la producción de posible uso en este campo debido a sus bajos empleos a aquellas familias que se dedican valores de combustión que hace menores los a la agricultura brindando oportunidades riesgos de quema; obteniendo por medio de de desarrollarse y tener un mejor estilo de esto una metodología de sus aplicaciones vida, El bambú es un material usado desde al postensado para elaborar estructuras la antigüedad, y actualmente su contribución sostenibles que aporten un buen uso de los crece en importancia, aumentando la materiales renovables que nos ofrece la comodidad y el bienestar del ser humano [12]. naturaleza como lo es el bambú o guadua angustifolia [8]. 
Materiales y métodos

Este proyecto de investigación va tener un enfoque cualitativo o también llamado categórico son características o atributos que se expresan de forma verbal (no numéricos), es decir, mediante palabras. Estas pueden ser: dicotómicas o policotomicas [13]; de la misma manera este trabajo está apoyado en una revisión documental, dado que está orientada en un proceso sistemático, riguroso, racional de recolección de datos y análisis de información obtenidos y procesados anteriormente por organismos oficiales, archivos, instituciones públicas o privadas, entre otras; la misma se realiza a nivel descriptivo ya que, describe y analiza las características y funciones del misma, a partir del análisis de estos datos secundarios se puede elaborar importantes conclusiones relacionadas con el comportamiento o estado de las variables.

Tabla 1

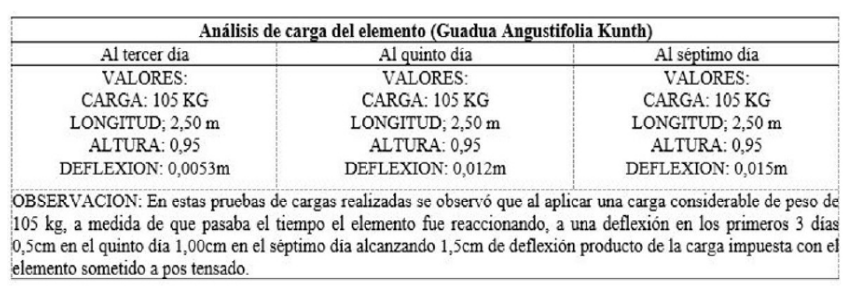

Tabla 2

\begin{tabular}{|c|c|c|}
\hline \multicolumn{3}{|c|}{ Análisis de carga del elemento (Guadua Angustifolia Kunth) } \\
\hline Al tercer dia & Al quinto dia & Al séptimo día \\
\hline VALORES: & VALORES: & VALORES: \\
CARGA: $105 \mathrm{KG}$ & CARGA: $105 \mathrm{KG}$ & CARGA: $105 \mathrm{KG}$ \\
LONGITUD; $2,50 \mathrm{~m}$ & LONGITUD; $2,50 \mathrm{~m}$ & LONGTUD; $; 2,50 \mathrm{~m}$ \\
ALTURA: 0,95 & ALTURA: 0,95 & ALTURA: 0,95 \\
DEFLEXION: $0,018 \mathrm{~m}$ & DEFLEXION: $0,033 \mathrm{~m}$ & DEFLEXION: $0,046 \mathrm{~m}$ \\
\hline OBSERVACIÓN: en estas pruebas de cargas realizadas se observó que al aplicar una carga considerable de peso de \\
$105 \mathrm{~kg}$, a medida de que pasaba el tiempo el elemento fue reaccionando, a una deflexión en los primeros 3 dias \\
$1,8 \mathrm{~cm}$ en el quinto dia $3,3 \mathrm{~cm}$ en el séptimo día alcanzando $4,6 \mathrm{~cm}$ de deflexión producto de la carga impuesta con e) \\
elemento sometido sin pos tensado.
\end{tabular}

Tabla 3

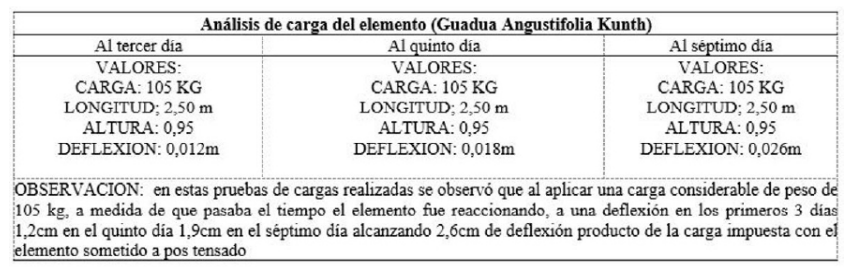

Tabla 4

\begin{tabular}{|c|c|c|}
\hline \multicolumn{3}{|c|}{ Análisis de carga del elemento (Guadua Angustifolia Kunth) } \\
\hline Al tercer dia & Al quinto dia & Al séptimo dia \\
\hline VALORES: & VALORES: & VALORLS: \\
\hline CARGA: $105 \mathrm{KG}$ & $105 \mathrm{KG}$ & CARGA: $105 \mathrm{KG}$ \\
\hline LONGITUD; $2,50 \mathrm{~m}$ & LONGITUD; $2,50 \mathrm{~m}$ & LONGITUD; $2,50 \mathrm{~m}$ \\
\hline ALTURA: 0,95 & & ALTURA: 0,95 \\
\hline DEFLEXION: $0.021 \mathrm{~m}$ & DEFLEXION: $0.034 \mathrm{~m}$ & DEFLEXION: $0.047 \mathrm{~m}$ \\
\hline \multicolumn{3}{|c|}{$\begin{array}{l}\text { OBSERVACION: en estas pruebas de cargas realizadas se observó que al aplicar una carga considerable de peso } \\
105 \mathrm{~kg} \text {, a medida de que pasaba el tiempo el elemento fue reaccionando, a una deflexiôn en los primeros } 3 \text { di } \\
2,1 \mathrm{~cm} \text { en el quinto dia } 3.4 \mathrm{~cm} \text { en el séntimo dia alcanzando } 4.7 \mathrm{~cm} \text { de deflexión producto de la carga impuresta con } \\
\text { elemento sometido sin pos tensado }\end{array}$} \\
\hline
\end{tabular}

En esta aplicación de la guadua pos tensada y sin pos tensar se utilizó los siguientes materiales: [14] Guadua angustifolia Kunth de dos distintas clases una (seca o madura) de utilización del tipo de construcción y otras verdes (recién cortadas y sin madurar) también se utilizó unos anillos de diámetro igual a la guadua utilizada y guaya $y$ tornillos para pos tensar, además de estos materiales se utilizaron herramientas como sierra, destornillador, taladro, alicate, que nos sirvieron de gran ayuda para ensamblar nuestra guadua y aplicarle las cargas de 105 $\mathrm{kg}$ por 7 días lo que nos dio unos resultados que nos permite decir que al someter la guadua a pos tensado adquiere más resistencia a la carga sometida y su deflexión es menor que la obtenida con la guadua sin pos tensar, también hubieron grandes diferencias al aplicar la carga a la guadua sin madurar (recién cortada) donde nos demuestra que sin un proceso de maduración y secado no se obtiene las mismas cualidades al someter está a cargas iguales [15].

\section{Conclusiones}

Se observó que la guadua al ser sometida a pos tensado sin maduración no resistió lo mismo que la guadua madura, ya que al no estar madura sus propiedades mecánicas no son las mismas y por esto resiste menos y su deflexión mayor. Se pudo determinar que al obtener datos de la guadua sin madura y seca al pos tensado resiste más deflexión que la guadua sin pos tensar ya que sus fibras obtienen mayor resistencia al ser apretadas y le aporta mejor comportamiento al momento de someter el elemento a carga. Se podría 


\section{4}

decir que este material es muy bueno pero se requiere de más pruebas para saber si podría soportar cargas de la magnitud de un vehículo para así determinar si se puede emplear en puentes vehiculares.

\section{Referencias}

[1] D.M. Moreno y S.I Brugger-Jakob. "Aprovechamiento del Bambú para impulsar el desarrollo económico sustentable en México", Observatorio de la Economía Latinoamericana, no. 176, 2012. [En línea]. Disponible en: http:// www.eumed.net/cursecon/ecolat/mx/2012/ desarrollo-economico-sustentable-mexico. html

[2] E.M. Moncada-Bustamante y M.S. RodríguezReyes, "Potencial económico de los cañaverales de Bambú de lazona 5 del Ecuador en el comercio de Emisiones", tesis de pregrado, Universidad de Guayaquil, Guayaquil, Ecuador, 2018

[3] O. De Luna-Bugallo, "Desarrollo de la comunidad de Hueytamalco Puebla México a través del bambú como materia industrial", tesis de maestría, Universidad Autónoma de Nuevo León, México, 2014

[4] C.M. Herrera-Mesén, "y diseño estructural de una vivienda construida a base de bambú", tesis de pregrado, Instituto Tecnológico de Costa Rica, Cartago, Costa Rica, 2008

[5] O. Jaramillo-Gómez, "La Formación Socioeconómica de Caldas y sus características Políticas". Antropol.sociol. no. 11, pp. 229 253, enero - diciembre 2009.

[6] F.V. Armendáriz- Carranza, J.A. TacoÁlvarez, "Cálculo y diseño estructural para la cubierta del mercado central de la parroquia de Píntag en base a tenso-estructura con el uso de bambú gigante (dendrocalamus asper)", tesis de pregrado. Escuela Politécnica del Ejército, Ecuador.

[7] Y. Contreras-Valero, Y. Cardona-Rodríguez, y D. A. Torres-Sánchez, "Estudio térmico e infrarrojo de cinco tipos de madera comercializadas en norte de santander, para su posible uso como fuente de energía", Respuestas, vol. 22, no. 2, pp. 36-47, jul. 2017

[8] X. Londoño. El bambú en Colombia. Sociedad Colombiana del Bambú. Biotecnología Vegetal, vol. 11, no. 3, pp. 143 - 154, julio - septiembre, 2011

[9] L. González, "Comportamiento a flexión de la Guadua Angustifolia con refuerzo pretensado", tesis de pregrado. Universidad Nacional de Colombia, 2003.

[10]E. De Navas-Gutiérrez y M.E. Herrero, "Aplicaciones estructurales de la Guadua. (Guadua Angustifolia Kunth). Proyecto de Estructura Modular Multifuncional en Colombia", tesis de pregrado, universidad Politécnica de Madrid. Madrid, España, 2011

[11] P. Luna, T. Caori, G. Granados, F. Lamus y Jorge Lozano, "Metodología de Diseño de Estructuras en Guadua Angustifolia como Material Estructural por el Método de Esfuerzos Admisibles", Revista en educación en ingeniería. no. 11, pp 66-75, 2011.

[12] J.C. Nolivos-Valiente y J.G. Yacelga-Díaz "Estudio de conexiones entre elementos estructurales de caña guadua sometidos a carga axial", tesis de pregrado. Escuela Politécnica Nacional, Pichincha, Ecuador,2010

[13] F. Arias, El proyecto de investigación: Introducción a la metodología científica. 5ta Ed. Caracas: Episteme, 2006 
[14] M. Añazco, Estudio de Vulnerabilidad del bambú. (Guadua Angustifolia) al cambio Climático en la costa del Ecuador y norte del Perú. Perú: IMBAR, 2013

[15] S. Vélez. La Guadua Angustifolia "El Bambú Colombiano", Símbolo y búsqueda de lo primitivo pp. 34-81, (s.f) 